\title{
Personalisierte Medizin - das Rad wurde neu erfunden
}

\author{
Karin Kraft \\ Lehrstuhl für Naturheilkunde, Medizinische Fakultät, Universität Rostock, Deutschland
}

Die personalisierte Medizin ist seit einigen Jahren eines der wichtigsten aktuellen Forschungsthemen der westlichen Medizin. Während die Entwicklung von individuellen tumorspezifischen Therapien einschließlich begleitender diagnostischer Gentests und noch umfassenderer «omics»-basierter Versuche, Diagnostik und Therapie zu personalisieren, ständig vorangetrieben wird, stehen neuerdings auch andere Aspekte wie z. B. die Verlängerung der beschwerdefreien Lebensspanne im Alter durch personalisierte Medizin im Fokus. Hintergrund ist die recht neue Erkenntnis, dass es in jeder Hinsicht lohnender wäre, den Prozess des Alterns (Seneszenz) selbst zu verlangsamen, um eine verlängerte gesunde Lebensspanne bis zum zehnten Lebensjahrzehnt und infolgedessen eine Kompression der Periode der altersassoziierten Erkrankungen zu erreichen, als diese Krankheiten selbst zu eliminieren. Dadurch würde sich die Lebensspanne nur um wenige Jahre verlängern [1]. Dieses Vorgehen scheint übrigens auch eine der wenigen Möglichkeiten zu sein, die derzeit explodierenden Kosten im Gesundheitswesen in vorhersehbarer Weise zu senken, denn die kostenintensive Phase der Multimorbidität würde damit auf eine relativ kurze Zeitspanne im sehr hohen Alter verlegt. Jedenfalls wurde in der Arbeit von Goldman et al. [1] in einer Modellrechnung für ältere US-Amerikaner ermittelt, dass eine Verzögerung der Seneszenz die Lebenserwartung um zusätzliche 2,2 Jahre steigern könnte, die vorwiegend bei guter Gesundheit verbracht würden. Der ökonomische Nutzen wurde - bei entsprechender Anhebung des Rentenalters - auf 7,1 Billionen USD für die nächsten 50 Jahre geschätzt. Im Gegensatz dazu würden die gegenwärtig üblichen, eher reparaturorientierten Strategien, die auf die separate Bekämpfung von kardiovaskulären und neurodegenerativen Erkrankungen sowie Krebs gerichtet sind, zu einer Abnahme der Fortschritte bei Gesundheit und Langlebigkeit führen, insbesondere wegen kompetitiver Risiken. Hier wäre die Adaptation für die Anwendung in einer hochaltrigen Patientenpopulation erforderlich.

Die Altersforschung der personalisierten Medizin wird zukünftig auf 3 Säulen basieren:

\section{KARGER}

Fax +4976145207 14

Information@Karger.com

www.karger.com
(C) 2014 S. Karger GmbH, Freiburg $1661-4119 / 14 / 0213-0152 \$ 39.50 / 0$

Accessible online at:

www.karger.com/fok
1. Ausbau des auf den bisherigen Kohortenstudien basierenden Wissens, wobei wegen der erforderlichen Differenzierung und Klassifikation der Patienten innerhalb einer Kohorte, z.B. entsprechend ihres Genotyps, sehr hohe Fallzahlen erforderlich sind und eine Studiendauer über Jahrzehnte notwendig ist.

2. Vergleichbare Studien bei geeigneten Tiermodellen, die den Vorteil haben, in wesentlich kürzeren Zeiträumen Ergebnisse zu erzielen.

3. Gezielte Untersuchungen zur Biologie des Alterns bei Mensch und Tier, um die Bedeutung von Biomarkern und den Wert von Interventionen abschätzen zu können. Dies schließt die Wirkungen von Genotyp und Umwelt und die Wirkungen der Interaktion zwischen verschiedenen Mechanismen sowie der Kombination von Interventionen ein.

Dafür wird ein sehr hoher Einsatz von Forschungsmitteln erforderlich sein, der auch die Kosten für das Management großer Datenmengen einschließt.

Wie ein Positionspapier von Anderson und Weindruch [2] zeigt, ist in den letzten Jahren neben der individuell adaptierten Bewegung das Konzept der Kalorienrestriktion zur möglichen Verlangsamung der Seneszenz von besonderem Interesse geworden, da diese nicht nur die Größe der Fettdepots reduziert, sondern auch die Expression von Schlüsselgenen, die am Energiestoffwechsel beteiligt sind, induziert und die Expression von über 50 proinflammatorischen Genen vermindert [3, 4].

In der Literatur finden sich diverse kleinere Studien mit einer Studiendauer von 6-12 Monaten. Bei adipösen Gesunden wurden Studien zur Kalorienrestriktion um bis zu 20\% durchgeführt [5-7]. Der Gewichtsverlust wurde von einer 24\%igen Abnahme des Körperfetts einschließlich einer 27\%igen Reduktion des Viszeralfetts begleitet [8]. In anderen Studien ergaben sich günstige Veränderungen der Serumkonzentrationen bei kardiovaskulären Risikofaktoren und der Insulinsensitivität [9-11]. Wie zu erwarten, war die Adhärenz in einigen Studien über die letzten Behandlungs- 
monate deutlich reduziert. Unklar ist auch, ob die günstigen Wirkungen der Kalorienreduktion bei Normalgewichtigen zu erzielen sind.

In Langzeitstudien (im Mittel über 6 Jahre Kalorienrestriktion) fanden sich ebenfalls reduzierte Serumwerte für Triglyzeride, Nüchternglukose und -insulin sowie niedrigere Blutdruckwerte im Vergleich zu den gematchten Kontrollen $[9,12]$.

$\mathrm{Ob}$ sich beim Menschen durch eine langfristige Kalorienrestriktion auch die Lebensspanne verlängern lässt, ist bisher unklar. Für andere Spezies, einschließlich die nicht-humanen Primaten, konnte dies bereits gezeigt werden [13]. Ganz in Sinne der personalisierten Medizin scheinen Wirksamkeit und Verträglichkeit einer restriktiven Kalorienzufuhr allerdings vom jeweiligen Genotyp und von verschiedenen Umweltfaktoren abzuhängen [14-17]. Die unfreiwillige hypokalorische Ernährung, die in vielen Ländern der Erde prävalent ist, wird übrigens fast immer von Malnutrition begleitet und hat jedenfalls (wohl deshalb) keinen lebensverlängernden Effekt.

Nun ist für die meisten Menschen eine langfristige Kalorienrestriktion ziemlich unattraktiv. Deshalb versucht man neuerdings, Nahrungsergänzungsmittel und Arzneimittel wie z.B. Resveratrol, Rapamycin oder Metformin zu verwenden, von denen bekannt ist, dass sie die Wirkung einer Kalorienrestriktion imitieren. Hierzu werden gegenwärtig Untersuchungen bei Mäusen im Hinblick auf die Verzögerung von Alterserkrankungen und die Verlängerung der maximalen Lebensspanne durchgeführt [2].

Moderate Modifikationen von Bewegungs- und Ernährungsmustern werden von vielen Menschen adhärent durchgeführt, wenn sie deren günstige Wirkung selbst spüren können. Dies gilt jedoch nicht für Nutraceuticals oder Medikamente, deren regelmäßige Einnahme deshalb weitaus schwieriger zu gewährleisten ist. Ihre Anwendung muss deshalb wissenschaftlich besonders gut abgesichert sein, auch im Hinblick auf mögliche Nebenwirkungen bei der Langzeiteinnahme. Die dafür erforderlichen Interventionsstudien sind sehr zeitaufwändig und sollten deshalb mit tierexperimentellen und In-vitro-Studien gekoppelt werden, die eine möglichst weitgehende Translation der Ergebnisse auf den Menschen ermöglichen. Hierbei muss jedoch zudem bedacht werden, dass der Alterungsprozess auf den verschiedenen Niveaus der Zellen, des Gewebes und der Organe sehr unterschiedlich ablaufen kann [18]. Es wird also noch länger dauern, bis die Optimierung der Energiebilanz mittels adäquater Ernährung, Einnahme von Nutraceuticals und körperlicher Aktivität im Sinne der personalisierten Medizin gelingen kann.

Wahrscheinlich haben Sie sich beim Lesen allmählich gefragt, wo denn hier die Komplementärmedizin bleibt. Genau darum geht es mir hier:Wenn Sie die von mir angegebene Literatur lesen, finden Sie keinerlei Hinweise auf Untersuchungen zum (Heil-)Fasten, auf naturheilkundliche Ernährungsformen und auf andere komplementärmedizinische Methoden. Ich habe diese Publikationen natürlich nicht unter dem Aspekt ausgewählt, dass sie derartige Hinweise nicht enthalten, sondern wegen ihrer Inhalte und Aussagen. Leider ist das ein ganz allgemeines Phänomen: Diese etablierten und von der Bevölkerung global gut angenommenen Methoden werden offenbar von der Scientific Community ignoriert, obwohl hier im letzten Jahrzehnt viele gut platzierte und qualifizierte Publikationen erschienen sind, in denen moderne Messparameter verwendet wurden und in denen gezeigt werden konnte, dass komplementäre Methoden nicht nur wirksam und dabei sehr gut verträglich sind, sondern dass man damit auch wirtschaftlich arbeiten kann. Auch die im vorliegenden Heft veröffentlichten Untersuchungen gehören dazu. Für mich jedenfalls gehört zu einer korrekten wissenschaftlichen Arbeitsweise, dass man Einseitigkeit vermeidet. Das ist auch im Sinne der Patienten.

\section{Literatur}

1 Goldman DP, Cutler D, Rowe JW, et al.: Substantial health and economic returns from delayed aging may warrant a new focus for medical research. Health Aff (Millwood) 2013;32:1698-1705.

2 Anderson RM, Weindruch R: The caloric restriction paradigm: implications for healthy human aging. Am J Hum Biol 2012;24:101-106.

3 Higami Y, Barger JL, Page GP, et al.: Energy restriction lowers the expression of genes linked to inflammation, the cytoskeleton, the extracellular matrix, and angiogenesis in mouse adipose tissue. $\mathrm{J}$ Nutr 2006;136:343-352.

4 Higami Y, Pugh TD, Page GP, et al.: Adipose tissue energy metabolism: altered gene expression profile of mice subjected to long-term caloric restriction. FASEB J 2004;18:415-417.

5 Weiss EP, Racette SB, Villareal DT, et al.: Improvements in glucose tolerance and insulin action induced by increasing energy expenditure or decreasing energy intake: a randomized controlled trial. Am J Clin Nutr 2006;84:1033-1042.
6 Heilbronn LK, de Jonge L, Frisard MI, et al.: Effect of 6-month calorie restriction on biomarkers of longevity, metabolic adaptation, and oxidative stress in overweight individuals: a randomized controlled trial. JAMA 2006;295:1539-1548.

7 Racette SB, Weiss EP, Villareal DT, et al.: One year of caloric restriction in humans: feasibility and effects on body composition and abdominal adipose tissue. J Gerontol A Biol Sci Med Sci 2006;61:943-950.

8 Redman LM, Heilbronn LK, Martin CK, Alfonso A, Smith SR, Ravussin E: Effect of calorie restriction with or without exercise on body composition and fat distribution. J Clin Endocrinol Metab 2007; 92:865-872.

9 Fontana L, Klein S: Aging, adiposity, and calorie restriction. JAMA 2007;297:986-994.

10 Lefevre M, Redman LM, Heilbronn LK, et al.: Caloric restriction alone and with exercise improves CVD risk in healthy non-obese individuals. Atherosclerosis 2009;203:206-213.

11 Larson-Meyer DE, Newcomer BR, Heilbronn LK, et al.: Effect of 6-month calorie restriction and exercise on serum and liver lipids and markers of liver function. Obesity (Silver Spring) 2008;16:1355-1362.
12 Meyer TE, Kovacs SJ, Ehsani AA, et al.: Long-term caloric restriction ameliorates the decline in diastolic function in humans. J Am Coll Cardiol 2006;47: 398-402.

13 Colman RJ, Anderson RM, Johnson SC, et al.: Caloric restriction delays disease onset and mortality in rhesus monkeys. Science 2009;325:201-204.

14 Bartke A: Single-gene mutations and healthy ageing in mammals. Philos Trans R Soc Lond B Biol Sci 2011;366:28-34.

15 Johnson SC, Rabinovitch PS, Kaeberlein M: mTOR is a key modulator of ageing and age-related disease. Nature 2013;17:338-345.

16 Liao CY, Johnson TE, Nelson JF: Genetic variation in responses to dietary restriction - an unbiased tool for hypothesis testing. Exp Gerontol 2013;48:1025-1029.

17 Mattison JA, Roth GS, Beasley TM, et al.: Impact of caloric restriction on health and survival in rhesus monkeys from the NIA study. Nature 2012;13:318-321.

18 Horvath S: DNA methylation age of human tissues and cell types. Genome Biol 2013;14:R115. 